\title{
PARISIAN RUIN OF SELF-SIMILAR GAUSSIAN RISK PROCESSES
}

\author{
KRZYSZTOF DȨBICKI, ${ }^{*}$ University of Wroctaw \\ ENKELEJD HASHORVA** AND \\ LANPENG JI, ${ }^{* * * * *}$ University of Lausanne
}

\begin{abstract}
In this paper we derive the exact asymptotics of the probability of Parisian ruin for selfsimilar Gaussian risk processes. Additionally, we obtain the normal approximation of the Parisian ruin time and derive an asymptotic relation between the Parisian and the classical ruin times.

Keywords: Parisian ruin time; Parisian ruin probability; self-similar Gaussian process; fractional Brownian motion; normal approximation; generalized Pickands' constant

2010 Mathematics Subject Classification: Primary 60G15

Secondary 60G70
\end{abstract}

\section{Introduction}

Let $\left\{X_{H}(t), t \geq 0\right\}$ be a centred self-similar Gaussian process with almost surely continuous sample paths and index $H \in(0,1)$, i.e. $\operatorname{var}\left(X_{H}(t)\right)=t^{2 H}$ and for any $a>0$ and $s, t \geq 0$,

$$
\operatorname{cov}\left(X_{H}(a t), X_{H}(a s)\right)=a^{2 H} \operatorname{cov}\left(X_{H}(t), X_{H}(s)\right) .
$$

Let $\beta, c$ be two positive constants. In risk theory, the surplus process of an insurance company can be modelled by

$$
R_{u}(t)=u+c t^{\beta}-X_{H}(t) \text { for } t \geq 0,
$$

where $u$ is the so-called initial reserve, $c t^{\beta}$ models the total premium received up to time $t$, and $X_{H}(t)$ represents the total amount of aggregated claims (including fluctuations) up to time $t$. Typically, classical risk models assume a linear premium income, meaning that $\beta=1$. In this paper we deal with a more general $\beta>H$ case allowing for a nonlinear premium income. Below we shall refer to $R_{u}$ as the self-similar Gaussian risk process. The justification for choosing self-similar processes to model the aggregated claim process comes from [35], where it was shown that the ruin probability for a self-similar Gaussian risk process is a good approximation of the ruin probability for some classical risk process. Recent contributions have shown that self-similar Gaussian processes such as fractional Brownian motion (fBm), sub-fractional Brownian motion (sub-fBm), and bi-fractional Brownian motion (bi-fBm) are useful in the modelling of financial risks, see, e.g. [19], [26]-[28], [31], and the references therein.

For any $u \geq 0$, define the classical ruin time of the self-similar Gaussian risk process by

$$
\tau_{u}=\inf \left\{t \geq 0: R_{u}(t)<0\right\} \quad \text { with } \inf \{\varnothing\}=\infty
$$

Received 13 June 2014; revision received 8 October 2014.

* Postal address: Mathematical Institute, University of Wrocław, pl. Grunwaldzki 2/4, 50-384 Wrocław, Poland.

** Postal address: University of Lausanne, UNIL-Dorigny, 1015 Lausanne, Switzerland.

*** Email address: jilanpeng@126.com 
and, thus, the probability of ruin is defined as

$$
\mathbb{P}\left\{\tau_{u}<\infty\right\}
$$

The classical ruin time and the probability of ruin for self-similar Gaussian risk processes are well studied in the literature; see, e.g. [17], [27], and [28].

Recently, an extension of the classical notion of ruin, i.e. the Parisian ruin, was the focus of substantial interest; see [6], [9], [10], and the references therein. The core notion of the extension to Parisian ruin is that now one allows the surplus process to spend a prespecified time under the level 0 before the ruin is recognized. To be more precise, let $T_{u}$ model the prespecified time which is a positive deterministic function of the initial reserve $u$. In our setup, the Parisian ruin time of the self-similar Gaussian risk process $R_{u}$ is defined as

$$
\tau_{u}^{*}=\inf \left\{t \geq T_{u}: t-\kappa_{t, u} \geq T_{u}\right\} \quad \text { with } \kappa_{t, u}=\sup \left\{s \in[0, t]: R_{u}(s) \geq 0\right\}
$$

Here, we use the convention that $\sup \{\varnothing\}=0$.

In this paper we focus on the Parisian ruin probability, i.e.

$$
\mathbb{P}\left\{\tau_{u}^{*}<\infty\right\}=\mathbb{P}\left\{\inf _{t \geq 0} \sup _{s \in\left[t, t+T_{u}\right]} R_{u}(s)<0\right\} ;
$$

see [5]-[7], [10], and [33] for a recent analysis of (1.2) for the Lévy surplus model. In mathematical finance, Parisian stopping times have been studied initially by Chesney et al. [4] in the context of barrier options.

Assume for the moment that $X_{H}$ is a standard Brownian motion, $\beta=1$, and $T_{u}=T>0$, $u>0$. Thus, $R_{u}$ is the Brownian motion risk process with a linear trend. As shown in [33], for any $u \geq 0$,

$$
\mathbb{P}\left\{\tau_{u}^{*}<\infty\right\}=\frac{\exp \left(-c^{2} T / 2\right)-c \sqrt{2 \pi T} \Phi(-c \sqrt{T})}{\exp \left(-c^{2} T / 2\right)+c \sqrt{2 \pi T} \Phi(c \sqrt{T})} \exp (-2 c u),
$$

where $\Phi(\cdot)$ is the distribution function of a standard normal random variable. Since the $\beta \neq 1$ case seems to be completely untractable, even for the Brownian motion risk process, one has to resort to bounds and asymptotic results, allowing the initial capital $u$ to become large; see, e.g. [20].

This paper is concerned with the asymptotic behaviour of the Parisian ruin probability as $u \rightarrow \infty$ for a large class of self-similar Gaussian risk processes. Under a local stationarity condition on the correlation of the self-similar process $X_{H}$ (see (2.4)) and a mild condition on $T_{u}$ (see (3.2)), in Theorem 3.1 we derive the asymptotics of the Parisian ruin probability. Interestingly, as a corollary, it appears that for the fBm risk process with a linear trend, if $H>\frac{1}{2}$,

$$
\mathbb{P}\left\{\tau_{u}^{*}<\infty\right\}=\mathbb{P}\left\{\tau_{u}<\infty\right\}(1+o(1)) \quad \text { as } u \rightarrow \infty,
$$

even if $T_{u}$ grows to $\infty$ at a specified rate as $u \rightarrow \infty$.

The combination of (1.4) with the asymptotic behaviour of $\mathbb{P}\left\{\tau_{u}<\infty\right\}$ derived in [27] thus implies the exact asymptotic behaviour of the Parisian ruin probability.

Additionally, we derive the approximation of the conditional (scaled) Parisian ruin time, and the asymptotic relation between the classical ruin time and the Parisian ruin time, given that the Parisian ruin occurs. This result is in agreement with, e.g. [2], [14], [20]-[24], [28], 
[29], and [36], where the approximation of the classical ruin time is considered. The obtained normal approximation of the Parisian ruin time is a new result, even for the Brownian motion risk process with a linear trend.

A brief outline of the paper. In Section 2 we introduce our notation and present a preliminary result concerning the tail of the sup-inf functional of a Gaussian random field. The asymptotics of the Parisian ruin probability is presented in Section 3, while the time of the Parisian ruin is presented in Section 4. Proofs are relegated to Section 5.

\section{Notation and preliminaries}

Let $\left\{X_{H}(t), t \geq 0\right\}$ be a centred self-similar Gaussian process with almost surely continuous sample paths and index $H \in(0,1)$ as defined in the introduction. By $\left\{B_{\alpha}(t), t \geq 0\right\}$, we denote a standard fBm with Hurst index $\alpha / 2 \in(0,1]$.

It is useful to define, for $\beta>H$ and $c>0$,

$$
Z(t)=\frac{X_{H}(t)}{1+c t^{\beta}} \quad \text { for } t \geq 0 .
$$

Indeed, by the self-similarity of $X_{H}$, for any positive $u$,

$$
\begin{aligned}
\mathbb{P}\left\{\tau_{u}^{*}<\infty\right\} & =\mathbb{P}\left\{\sup _{t \geq 0} \inf _{s \in\left[t, t+T_{u}\right]}\left(X_{H}(s)-c s^{\beta}\right)>u\right\} \\
& =\mathbb{P}\left\{\sup _{t \geq 0} \inf _{s \in\left[0, T_{u} u^{-1 / \beta}\right]} Z(t+s)>u^{1-H / \beta}\right\} .
\end{aligned}
$$

It follows that (cf. [27] and [28]) $\sigma_{Z}(t)=\sqrt{\operatorname{var}(Z(t))}$ attains its maximum on $[0, \infty)$ at the unique point

$$
t_{0}=\left(\frac{H}{c(\beta-H)}\right)^{1 / \beta}
$$

and

$$
\sigma_{Z}(t)=A-\frac{B A^{2}}{2}\left(t-t_{0}\right)^{2}+o\left(\left(t-t_{0}\right)^{2}\right) \quad \text { as } t \rightarrow t_{0},
$$

where

$$
A=\frac{\beta-H}{\beta}\left(\frac{H}{c(\beta-H)}\right)^{H / \beta}, \quad B=\left(\frac{H}{c(\beta-H)}\right)^{-(H+2) / \beta} H \beta .
$$

In the rest of the paper we assume the local stationarity of the standardized Gaussian process $\bar{X}_{H}(t):=X_{H}(t) / t^{H}, t>0$ in a neighbourhood of the point $t_{0}$, i.e.

$$
\lim _{s \rightarrow t_{0}, t \rightarrow t_{0}} \frac{\mathbb{E}\left(\left(\bar{X}_{H}(s)-\bar{X}_{H}(t)\right)^{2}\right)}{K^{2}(|s-t|)}=Q>0
$$

holds for some positive function $K(\cdot)$, which is assumed to be regularly varying at 0 with index $\alpha / 2 \in(0,1)$. Condition (2.4) is common in the literature; most of the known self-similar Gaussian processes (such as fBm, sub-fBm, and bi-fBm) satisfy (2.4); see, e.g. [25]. Note that the local stationarity at $t_{0}$ and the self-similarity of the process $X_{H}$ imply the local stationarity of $X_{H}$ at any point $r>0$, i.e.

$$
\lim _{s \rightarrow r, t \rightarrow r} \frac{\mathbb{E}\left(\left(\bar{X}_{H}(s)-\bar{X}_{H}(t)\right)^{2}\right)}{K^{2}(|s-t|)}=\left(\frac{t_{0}}{r}\right)^{\alpha} Q .
$$


Throughout this paper we denote by $K^{\leftarrow}(\cdot)$ the asymptotic inverse of $K(\cdot)$; by definition

$$
K^{\leftarrow}(K(t))=K\left(K^{\leftarrow}(t)\right)(1+o(1))=t(1+o(1)) \quad \text { as } t \rightarrow 0 .
$$

It follows that $K^{\leftarrow}(\cdot)$ is regularly varying at 0 with index $2 / \alpha$; see, e.g. [20].

Let $\mathscr{H}_{\alpha}$ be the classical Pickands' constant defined by

$$
\mathscr{H}_{\alpha}=\lim _{T \rightarrow \infty} \frac{1}{T} \mathbb{E}\left(\exp \left(\sup _{t \in[0, T]}\left(\sqrt{2} B_{\alpha}(t)-t^{\alpha}\right)\right)\right) ;
$$

see [1], [3], [11], [12], [15], [16], [18], [34], and [39] for the basic properties of the Pickands' and related constants. A new constant that will appear in our findings below is defined as

$$
\mathcal{F}_{\alpha}(T)=\lim _{S \rightarrow \infty} \frac{1}{S} \mathbb{E}\left(\exp \left(\sup _{t \in[0, S]} \inf _{s \in[0, T]}\left(\sqrt{2} B_{\alpha}(t+s)-(t+s)^{\alpha}\right)\right)\right) \in(0, \infty)
$$

for any $T \in[0, \infty)$.

We conclude this section with a general result for the tail of the sup-inf functional applied to the Gaussian process $Z$. Recall that by $\Phi(\cdot)$ we denote the distribution function of a standard normal random variable. In order to simplify the notation, we shall set

$$
q=q(v):=K^{\leftarrow}\left(\frac{1}{v}\right) \text { for } v>0 .
$$

Theorem 2.1. Let $\{Z(t), t \geq 0\}$ be the centred Gaussian process given as in (2.1), and let $x_{i}(\cdot), i=1,2$ be two functions such that

$$
\lim _{v \rightarrow \infty} x_{i}(v)=x_{i}, \quad \lim _{v \rightarrow \infty} x_{i}(v) v^{-1 / 2}=0, \quad i=1,2
$$

for some $x_{1}, x_{2} \in \mathbb{R} \cup\{\infty\}$ satisfying $x_{2}>-x_{1}$. Furthermore, for all large $v$ denote $\Theta_{x_{1}, x_{2}}(v)=\left[t_{0}-x_{1}(v) v^{-1}, t_{0}+x_{2}(v) v^{-1}\right]$. Then, for any positive function $\lambda(\cdot)$ such that $\lim _{v \rightarrow \infty} \lambda(v)=\lambda \in[0, \infty)$, we have

$$
\begin{aligned}
\mathbb{P}\left\{\sup _{t \in \Theta_{x_{1}, x_{2}}(v)} \inf _{s \in[0, \lambda(v) q]} Z(t+s)>v\right\}= & \frac{\mathcal{F}_{\alpha}\left(D_{0} \lambda\right)}{\mathcal{H}_{\alpha}}\left(\Phi\left(A^{-1 / 2} B^{1 / 2} x_{2}\right)-\Phi\left(-A^{-1 / 2} B^{1 / 2} x_{1}\right)\right) \\
& \times \mathbb{P}\left\{\sup _{t \geq 0} Z(t)>v\right\}(1+o(1)) \text { as } v \rightarrow \infty,
\end{aligned}
$$

where $D_{0}=2^{-1 / \alpha} A^{-2 / \alpha} Q^{1 / \alpha}$, and $\mathcal{F}_{\alpha}(\cdot)$ defined in (2.5) is positive and finite.

The complete proof of Theorem 2.1 is given in Section 5 .

\section{Asymptotics of the Parisian ruin probability}

In this section we display the main result of the paper, which is the asymptotics of the Parisian ruin probability $\mathbb{P}\left\{\tau_{u}^{*}<\infty\right\}$ as $u \rightarrow \infty$ for the self-similar Gaussian risk model in (1.1).

First, we note that in light of the seminal paper [27],

$$
\mathbb{P}\left\{\tau_{u}<\infty\right\}=\left(\frac{A^{3 / 2-2 / \alpha} Q^{1 / \alpha} \mathscr{H}_{\alpha}}{2^{1 / \alpha} B^{1 / 2}}\right) \frac{u^{2 H / \beta-2}}{K^{\leftarrow}\left(u^{H / \beta-1}\right)} \exp \left(-\frac{u^{2(1-H / \beta)}}{2 A^{2}}\right)(1+o(1))
$$

holds as $u \rightarrow \infty$. In order to control the growth of $T_{u}$, we shall assume that

$$
\lim _{u \rightarrow \infty} \frac{T_{u} u^{-1 / \beta}}{K^{\leftarrow}\left(u^{H / \beta-1}\right)}=T \in[0, \infty)
$$


Theorem 3.1. Let $\left\{R_{u}(t), t \geq 0\right\}$ be the self-similar Gaussian risk process given as in (1.1) with $X_{H}$ satisfying (2.4) and $T_{u}, u>0$ satisfying (3.2). If $\tau_{u}^{*}$ denotes the Parisian ruin time of $R_{u}$ then

$$
\mathbb{P}\left\{\tau_{u}^{*}<\infty\right\}=\frac{\mathcal{F}_{\alpha}\left(D_{0} T\right)}{\mathscr{H}_{\alpha}} \mathbb{P}\left\{\tau_{u}<\infty\right\}(1+o(1)) \text { as } u \rightarrow \infty,
$$

where $D_{0}=2^{-1 / \alpha} A^{-2 / \alpha} Q^{1 / \alpha}$ with $\mathcal{F}_{\alpha}(T)$ defined in (2.5).

The proof of Theorem 3.1 is deferred to Section 5; it relies on the general result for the asymptotics of sup-inf functional of the Gaussian process $Z$, given in Theorem 2.1.

Remark 3.1. Observe that Pickands' constant $\mathscr{H}_{\alpha}=\mathcal{F}_{\alpha}(0)$ and $\mathscr{H}_{1}=1$ (cf. [39]). It is not clear how to calculate $\mathcal{F}_{\alpha}(T)$ using the definition in (2.5). However, for the $\alpha=1$ special case, (1.3) and (3.3) below imply that

$$
\mathcal{F}_{1}(T)=\frac{\exp (-T / 4)-\sqrt{\pi T} \Phi(-\sqrt{T / 2})}{\exp (-T / 4)+\sqrt{\pi T} \Phi(-\sqrt{T / 2})} \text { for } T>0 .
$$

In this paper we shall refer to $\mathcal{F}_{\alpha}(T)$ as the generalized Pickands' constant.

As a corollary of the last theorem, we next present a result for the fBm risk processes with a linear trend where $X_{H}$ is assumed to be a standard $\mathrm{fBm} B_{2 H}$. Specifically, for any $H \in(0,1]$, we have

$$
\operatorname{cov}\left(X_{H}(t), X_{H}(s)\right)=\frac{1}{2}\left(t^{2 H}+s^{2 H}-|t-s|^{2 H}\right) \quad \text { for } t, s \geq 0
$$

and, thus, (2.4) holds with $K(t)=t^{H}, t \geq 0$, and $Q=t_{0}^{-2 H}=[H /(c(\beta-H))]^{-2 H / \beta}$ if, further, $\beta>H$.

Corollary 3.1. Let $R_{u}(t)=u+c t-B_{2 H}(t), t \geq 0$, and let $T_{u}, u>0$ be such that $\lim _{u \rightarrow \infty} T_{u} u^{1 / H-2}=T \in[0, \infty)$. If $c>0$ and $H \in(0,1)$ then

$$
\begin{aligned}
\mathbb{P}\left\{\tau_{u}^{*}<\infty\right\}= & \mathcal{F}_{2 H}\left(D_{0} T\right) \frac{2^{-1 / 2 H}}{\sqrt{H(1-H)}}\left(\frac{c^{H} u^{1-H}}{H^{H}(1-H)^{1-H}}\right)^{1 / H-2} \\
& \times \exp \left(-\frac{c^{2 H} u^{2(1-H)}}{2 H^{2 H}(1-H)^{2(1-H)}}\right)(1+o(1)) \text { asu } \rightarrow \infty,
\end{aligned}
$$

where $D_{0}=2^{-1 / 2 H} c^{2} H^{-2}(1-H)^{2-1 / H}$.

Remark 3.2. Using the fact that $\mathcal{F}_{2 H}(0)=\mathscr{H}_{2 H}$, Corollary 3.1 implies that

$$
\mathbb{P}\left\{\tau_{u}^{*}<\infty\right\}=\mathbb{P}\left\{\tau_{u}<\infty\right\}(1+o(1)) \quad \text { as } u \rightarrow \infty
$$

if $T=0$ (i.e. $T_{u}=o\left(u^{(2 H-1) / H}\right)$ ). Thus, if $H>\frac{1}{2}$, the asymptotics of the Parisian ruin probability coincide with the asymptotics of the classical ruin probability even if $T_{u}$ grows to $\infty$, provided that $T=0$. This property is another manifestation of the long-range dependence structure of fBm with Hurst index $H>\frac{1}{2}$.

For the $T_{u}=T u^{1 / H-2}$ boundary case with $T>0$, the Parisian ruin probability and the classical ruin probability are not asymptotically equivalent, as the initial capital $u$ tends to $\infty$.

In [32] a different type of Parisian ruin wass considered, where the deterministic prespecified time $T_{u}$ is replaced by an independent random variable (in particular, an exponential random variable is dealt with therein; see also [8]). In the following corollary we calculate the Parisian ruin probability of this model. 
Corollary 3.2. Let $\left\{R_{u}(t), t \geq 0\right\}$ be the self-similar Gaussian risk process given as in (1.1) with $X_{H}$ satisfying (2.4). If $\mathcal{T}$ is a positive random variable independent of $\left\{R_{u}(t), t \geq 0\right\}$, then

$$
\mathbb{P}\left\{\inf _{t \geq 0} \sup _{s \in[t, t+\mathcal{T}]} R_{u}(s)<0\right\}=\mathbb{P}\left\{\tau_{u}<\infty\right\}(1+o(1)) \text { as } u \rightarrow \infty
$$

holds, provided that $2 H+\alpha>2 \beta$.

\section{Normal approximation of the Parisian ruin time}

In this section we present a normal approximation for the conditional (scaled) Parisian ruin time. Additionally, we derive an asymptotic relation between the classical ruin time and the Parisian ruin time, given that the Parisian ruin occurs.

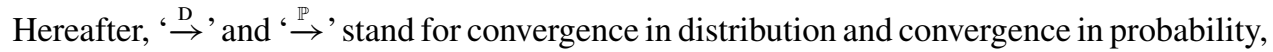
respectively.

Theorem 4.1. Let $\tau_{u}, \tau_{u}^{*}$ be the classical ruin time and the Parisian ruin time for the self-similar Gaussian risk process $\left\{R_{u}(t), t \geq 0\right\}$ given as in (1.1). If $X_{H}$ satisfies (2.4) and $T_{u}, u>0$ satisfies (3.2), then

$$
\frac{\tau_{u}^{*}-t_{0} u^{1 / \beta}}{A^{1 / 2} B^{-1 / 2} u^{H / \beta+1 / \beta-1}} \mid\left(\tau_{u}^{*}<\infty\right) \stackrel{\mathrm{D}}{\rightarrow} \mathcal{N} \quad \text { as } u \rightarrow \infty,
$$

where $A, B$ are as in (2.3) and $\mathcal{N}$ is a standard normal random variable. Moreover,

$$
\frac{\tau_{u}^{*}-\tau_{u}}{u^{H / \beta+1 / \beta-1}} \mid\left(\tau_{u}^{*}<\infty\right) \stackrel{\mathbb{P}}{\rightarrow} 0 \quad \text { as } u \rightarrow \infty .
$$

The complete proof of Theorem 4.1 is given in Section 5 .

As a straightforward implication of Theorem 4.1, it follows that if $H+1=\beta$ then

$$
\left(\tau_{u}^{*}-\tau_{u}\right) \mid\left(\tau_{u}^{*}<\infty\right) \stackrel{\mathbb{P}}{\rightarrow} 0 \quad \text { as } u \rightarrow \infty .
$$

Remark 4.1. In [28] a slightly more general class of Gaussian processes was considered. Under the additional technical conditions [28, A1 and A3] similar results as in Theorem 3.1 and Theorem 4.1 also hold for that class of Gaussian processes; the only difference is that in (4.1) and (4.2) we shall have $\sqrt{\operatorname{var}\left(X_{H}\left(u^{1 / \beta}\right)\right)}$ instead of $u^{H / \beta}$ and $s_{0}(u)$ (in their notation) instead of $t_{0}$.

We note that extensions of our result to Gaussian processes with random variance under similar conditions as in [30] are also possible.

\section{Proofs}

This section is dedicated to the proofs of Theorems 2.1, 3.1, 4.1, and Corollary 3.2. We first present a crucial lemma which can be seen as an extension of the celebrated Pickands' lemma; see, e.g. [37]-[39]. We refer to [13] for recent developments in this direction.

Let $\lambda_{1}, \lambda_{2}$ be two given positive constants. Consider a family of almost surely continuous centred Gaussian random fields

$$
\left\{X_{v}(t, s),(t, s) \in\left[0, \lambda_{1}\right] \times\left[0, \lambda_{2}\right]\right\}
$$


indexed by $v>0$. We shall assume that its variance equals 1 and the correlation functions $r_{v}\left(t, s, t^{\prime}, s^{\prime}\right)=\operatorname{cov}\left(X_{v}(t, s), X_{v}\left(t^{\prime}, s^{\prime}\right)\right),(t, s),\left(t^{\prime}, s^{\prime}\right) \in\left[0, \lambda_{1}\right] \times\left[0, \lambda_{2}\right], v>0$ satisfy the following two conditions.

Condition 5.1. There exist constants $D>0, \alpha \in(0,2]$, and a positive function $f(\cdot)$ defined in $(0, \infty)$ such that

$$
\lim _{v \rightarrow \infty}(f(v))^{2}\left(1-r_{v}\left(t, s, t^{\prime}, s^{\prime}\right)\right)=D\left|s+t-s^{\prime}-t^{\prime}\right|^{\alpha}
$$

holds for any $(t, s),\left(t^{\prime}, s^{\prime}\right) \in\left[0, \lambda_{1}\right] \times\left[0, \lambda_{2}\right]$.

Condition 5.2. There exist constants $C>0, v_{0}>0, \gamma \in(0,2]$ such that for any $v>v_{0}$ with $f(\cdot)$ given in Condition 5.1,

$$
(f(v))^{2}\left(1-r_{v}\left(t, s, t^{\prime}, s^{\prime}\right)\right) \leq C\left(\left|s-s^{\prime}\right|^{\gamma}+\left|t-t^{\prime}\right|^{\gamma}\right)
$$

holds uniformly with respect to $(t, s),\left(t^{\prime}, s^{\prime}\right) \in\left[0, \lambda_{1}\right] \times\left[0, \lambda_{2}\right]$.

Lemma 5.1. Let $\left\{X_{v}(t, s),(t, s) \in\left[0, \lambda_{1}\right] \times\left[0, \lambda_{2}\right]\right\}, v>0$ be the family of centred Gaussian random fields with variance equal to 1 , defined above. If both Condition 5.1 and Condition 5.2 hold, then for any positive function $\theta(\cdot)$ satisfying $\lim _{v \rightarrow \infty} f(v) / \theta(v)=1$, we have

$$
\begin{aligned}
& \mathbb{P}\left\{\sup _{t \in\left[0, \lambda_{1}\right]} \inf _{s \in\left[0, \lambda_{2}\right]} X_{v}(t, s)>\theta(v)\right\} \\
& \quad=\mathscr{H}_{\alpha}\left(D^{1 / \alpha} \lambda_{1}, D^{1 / \alpha} \lambda_{2}\right)(1+o(1)) \frac{1}{\sqrt{2 \pi} \theta(v)} \exp \left(-\frac{(\theta(v))^{2}}{2}\right) \text { as } u \rightarrow \infty,
\end{aligned}
$$

where

$$
\mathscr{H}_{\alpha}\left(\lambda_{1}, \lambda_{2}\right)=\mathbb{E}\left(\exp \left(\sup _{t \in\left[0, \lambda_{1}\right]} \inf _{s \in\left[0, \lambda_{2}\right]}\left(\sqrt{2} B_{\alpha}(t+s)-(t+s)^{\alpha}\right)\right)\right) \in(0, \infty) .
$$

Proof. Note that the sup-inf functional satisfies [13, Conditions F1 and F2]. The proof follows by similar arguments as the proof of Lemma 1 therein, and, therefore, we omit the technical details.

The next result plays an important role in the proof of Theorem 3.1; see [27] for its proof.

Lemma 5.2. Let $\{Z(t), t \geq 0\}$ be defined as in (2.1) and set $v(u)=u^{1-H / \beta}$. If $c>0$ and $\beta>H$, then for any $G>t_{0}$, we have

$$
\begin{aligned}
\mathbb{P}\left\{\tau_{u}<\infty\right\} & =\mathbb{P}\left\{\sup _{t \in[0, G]}\left(X_{H}(t)-c t^{\beta}\right)>u\right\}(1+o(1)) \\
& =\mathbb{P}\left\{\sup _{t \in\left[t_{0}-\ln v(u) / v(u), t_{0}+\ln v(u) / v(u)\right]} Z(t)>v(u)\right\}(1+o(1)) \quad \text { as } u \rightarrow \infty
\end{aligned}
$$

Furthermore,

$$
\mathbb{P}\left\{\sup _{\left|t-t_{0}\right|>\ln v(u) / v(u)} Z(t)>v(u)\right\}=o\left(\mathbb{P}\left\{\sup _{t \geq 0} Z(t)>v(u)\right\}\right) \quad \text { as } u \rightarrow \infty .
$$




\subsection{Proof of Theorem 2.1}

We shall provide only the proof for the $\infty>x_{2}>0>-x_{1}>-\infty$ case. The other cases can be established by similar arguments. Since our approach is of an asymptotic nature, we assume in the following that $v$ is sufficiently large so that $x_{i}(v)>0, i=1,2$. Let $S>2 \lambda$ be any positive constant. With $q=q(v)$ defined in (2.6), we denote

$$
\triangle_{k}=[k S q,(k+1) S q] \quad \text { for } k \in \mathbb{Z}, \quad N_{i}(v)=\left\lfloor S^{-1} x_{i}(v) q^{-1} v^{-1}\right\rfloor \quad \text { for } i=1,2,
$$

where $\lfloor\cdot\rfloor$ is the ceiling function. For any small $\varepsilon_{0}>0$, denote $\lambda_{\varepsilon_{0}}^{+}=\lambda+\varepsilon_{0}$ and $\lambda_{\varepsilon_{0}}^{-}=$ $\max \left(0, \lambda-\varepsilon_{0}\right)$. It follows by Bonferroni's inequality that

$$
\begin{aligned}
\sum_{k=-N_{1}(v)-1}^{N_{2}(v)+1} Q_{k}^{+}(v) & \geq \mathbb{P}\left\{\sup _{t \in \Theta_{x_{1}, x_{2}}(v)} \inf _{s \in[0, \lambda(v) q]} Z(t+s)>v\right\} \\
& \geq \sum_{k=-N_{1}(v)}^{N_{2}(v)} Q_{k}^{-}(v)-\Sigma_{1}(v)
\end{aligned}
$$

for large enough $u$, where

$$
\begin{array}{ll}
Q_{k}^{+}(v)=\mathbb{P}\left\{\sup _{t \in \triangle_{k}} \inf _{s \in\left[0, \lambda_{\varepsilon_{0}}^{-} q\right]} Z\left(t_{0}+t+s\right)>v\right\}, & k \in \mathbb{Z}, \\
Q_{k}^{-}(v)=\mathbb{P}\left\{\sup _{t \in \triangle_{k}} \inf _{s \in\left[0, \lambda_{\varepsilon_{0}}^{+} q\right]} Z\left(t_{0}+t+s\right)>v\right\}, & k \in \mathbb{Z},
\end{array}
$$

and

$$
\begin{array}{r}
\Sigma_{1}(v)=\sum_{-N_{1}(v) \leq k<l \leq N_{2}(v)} \mathbb{P}\left\{\sup _{t \in \triangle_{k}} \inf _{s \in\left[0, \lambda_{\varepsilon_{0}}^{+} q\right]} Z\left(t_{0}+t+s\right)>v,\right. \\
\left.\sup _{t \in \triangle_{l}} \inf _{s \in\left[0, \lambda_{\varepsilon_{0}}^{+} q\right]} Z\left(t_{0}+t+s\right)>v\right\} .
\end{array}
$$

Next, we shall derive upper bounds for $Q_{k}^{+}(v)$ and lower bounds for $Q_{k}^{-}(v)$. First, note that

$$
\begin{aligned}
& Q_{k}^{+}(v) \leq \mathbb{P}\left\{\sup _{t \in \triangle_{k}} \inf _{s \in\left[0, \lambda_{\varepsilon_{0}}^{-} q\right]} \bar{Z}\left(t_{0}+t+s\right)>\frac{v}{\sigma_{Z}^{+}(k, v)}\right\}, \\
& Q_{k}^{-}(v) \geq \mathbb{P}\left\{\sup _{t \in \triangle_{k}} \inf _{s \in\left[0, \lambda_{\varepsilon_{0}}^{+} q\right]} \bar{Z}\left(t_{0}+t+s\right)>\frac{v}{\sigma_{Z}^{-}(k, v)}\right\},
\end{aligned}
$$

where $\bar{Z}(t):=Z(t) / \sigma_{Z}(t), t \geq 0$ and

$$
\sigma_{Z}^{-}(k, v)=\inf _{t \in \triangle_{k}} \inf _{s \in\left[0, \lambda_{\varepsilon_{0}}^{+} q\right]} \sigma_{Z}\left(t_{0}+t+s\right), \quad \sigma_{Z}^{+}(k, v)=\sup _{t \in \triangle_{k}} \sup _{s \in\left[0, \lambda_{\varepsilon_{0}} q\right]} \sigma_{Z}\left(t_{0}+t+s\right) .
$$

Furthermore, since

$$
\sigma_{Z}(t)=A-\frac{A^{2} B}{2}\left(t-t_{0}\right)^{2}(1+o(1)) \quad \text { as } t \rightarrow t_{0}
$$


for any small $\varepsilon_{1}>0$, there exists $v_{0}$ such that for any $v>v_{0}$ (where we set $B^{ \pm}=B\left(1 \pm \varepsilon_{1}\right)$ ),

$$
\frac{1}{\sigma_{Z}^{-}(k, v)} \leq \frac{1}{A}+\frac{B^{+}}{2}\left(\left((k+1) S+\lambda_{\varepsilon_{0}}^{+}\right) q\right)^{2}, \quad \frac{1}{\sigma_{Z}^{+}(k, v)} \geq \frac{1}{A}+\frac{B^{-}}{2}(k S q)^{2}
$$

hold for $k=0, \ldots, N_{2}(v)+1$, and also

$$
\frac{1}{\sigma_{Z}^{-}(k, v)} \leq \frac{1}{A}+\frac{B^{+}}{2}(k S q)^{2}, \quad \frac{1}{\sigma_{Z}^{+}(k, v)} \geq \frac{1}{A}+\frac{B^{-}}{2}\left(\left((k+1) S+\lambda_{\varepsilon_{0}}^{-}\right) q\right)^{2}
$$

hold for $k=-N_{1}(v)-1, \ldots,-1$. Moreover, for any $k=-N_{1}(v)-1, \ldots, N_{2}(v)+1$, set $\bar{Z}_{k, v}(t, s)=\bar{Z}\left(t_{0}+k S q+t q+s q\right),(t, s) \in[0, S] \times\left[0, \lambda_{\varepsilon_{0}}^{+}\right]$. It follows from (2.4) that for the correlation function $r_{\bar{Z}_{k, v}}(\cdot, \cdot, \cdot, \cdot)$ of $\bar{Z}_{k, v}$,

$$
\lim _{v \rightarrow \infty} 2 v^{2}\left(1-r_{\bar{Z}_{k, v}}\left(t, s, t^{\prime}, s^{\prime}\right)\right)=Q\left|s+t-s^{\prime}-t^{\prime}\right|^{\alpha}
$$

holds for any $(t, s),\left(t^{\prime}, s^{\prime}\right) \in[0, S] \times\left[0, \lambda_{\varepsilon_{0}}^{+}\right]$. Furthermore, for sufficiently large $v$,

$$
2 v^{2}\left(1-r_{\bar{Z}_{k, v}}\left(t, s, t^{\prime}, s^{\prime}\right)\right) \leq G_{0} \frac{K^{2}\left(q\left|s+t-s^{\prime}-t^{\prime}\right|\right)}{K^{2}(q)}
$$

for all $(t, s),\left(t^{\prime}, s^{\prime}\right) \in[0, S] \times\left[0, \lambda_{\varepsilon_{0}}^{+}\right]$, with some positive constant $G_{0}$. Set

$$
S_{\max }=\max \left\{\left|s+t-s^{\prime}-t^{\prime}\right|:(t, s),\left(t^{\prime}, s^{\prime}\right) \in[0, S] \times\left[0, \lambda_{\varepsilon_{0}}^{+}\right]\right\} .
$$

Using Potter bounds (cf. [20]) for any small $\delta>0$, we have, when $v$ is sufficiently large,

$$
\begin{aligned}
\frac{K^{2}\left(q\left|s+t-s^{\prime}-t^{\prime}\right|\right)}{K^{2}(q)} & \leq G_{1} \max \left(S_{\max }^{\alpha-\delta}, S_{\max }^{\alpha+\delta}\right)\left(\frac{\left|s+t-s^{\prime}-t^{\prime}\right|}{S_{\max }}\right)^{\alpha-\delta} \\
& \leq G_{2}\left(\left|t-t^{\prime}\right|^{\alpha-\delta}+\left|s-s^{\prime}\right|^{\alpha-\delta}\right)
\end{aligned}
$$

holds uniformly with respect to $(t, s),\left(t^{\prime}, s^{\prime}\right) \in[0, S] \times\left[0, \lambda_{\varepsilon_{0}}^{+}\right]$, where $G_{1}, G_{2}$ are two positive constants. Hence, by an application of Lemma 5.1, where we set

$$
f(v)=\frac{v}{A}, \quad \theta_{k}(v)=\left(\frac{1}{A}+\frac{B^{+}}{2}\left(\left((k+1) S+\lambda_{\varepsilon_{0}}^{+}\right) q\right)^{2}\right) v, \quad D=\frac{Q}{2 A^{2}},
$$

we obtain, for any $k=0, \ldots, N_{2}(v)+1$,

$$
Q_{k}(v) \geq \mathscr{H}_{\alpha}\left(D_{0} S, D_{0} \lambda_{\varepsilon_{0}}^{+}\right) \frac{1}{\sqrt{2 \pi} \theta_{k}(v)} \exp \left(-\frac{\left(\theta_{k}(v)\right)^{2}}{2}\right)(1+o(1)) \quad \text { as } u \rightarrow \infty,
$$

where $D_{0}=D^{1 / \alpha}=2^{-1 / \alpha} A^{-2 / \alpha} Q^{1 / \alpha}$. Therefore, as $v \rightarrow \infty\left(\operatorname{set} \zeta(v)=v^{-2} q^{-1} \mathrm{e}^{-v^{2} / 2 A^{2}}\right)$,

$$
\begin{aligned}
\sum_{k=0}^{N_{2}(v)} Q_{k}(v) & \geq \mathscr{H}_{\alpha}\left(D_{0} S, D_{0} \lambda_{\varepsilon_{0}}^{+}\right) \frac{A}{\sqrt{2 \pi} v} \sum_{k=0}^{N_{2}(v)} \exp \left(-\frac{\left(\theta_{k}(v)\right)^{2}}{2}\right)(1+o(1)) \\
& =\frac{1}{S} \mathscr{H}_{\alpha}\left(D_{0} S, D_{0} \lambda_{\varepsilon_{0}}^{+}\right) \frac{A}{\sqrt{2 \pi}} \zeta(v) \int_{0}^{x_{2}} \exp \left(-\frac{B^{+}}{2 A} x^{2}\right) \mathrm{d} x(1+o(1)),
\end{aligned}
$$


where we used the fact that

$$
\lim _{v \rightarrow \infty} v q=\lim _{v \rightarrow \infty} v K^{\leftarrow}\left(\frac{1}{v}\right)=0, \quad \lim _{v \rightarrow \infty} x_{2}(v) v^{-1 / 2}=0 .
$$

Similarly, as $v \rightarrow \infty$,

$$
\sum_{k=-N_{1}(v)}^{-1} Q_{k}(v) \geq \frac{1}{S} \mathscr{H}_{\alpha}\left(D_{0} S, D_{0} \lambda_{\varepsilon_{0}}^{+}\right) \frac{A}{\sqrt{2 \pi}} \zeta(v) \int_{-x_{1}}^{0} \exp \left(-\frac{B^{+}}{2 A} x^{2}\right) \mathrm{d} x(1+o(1)) .
$$

Furthermore, with the same arguments as above for any $S_{1}>2 \lambda$,

$$
\begin{aligned}
\sum_{k=-N_{1}(v)-1}^{N_{2}(v)+1} Q_{k}(v) \leq & \frac{1}{S_{1}} \mathscr{H}_{\alpha}\left(D_{0} S_{1}, D_{0} \lambda_{\varepsilon_{0}}^{-}\right) \frac{A}{\sqrt{2 \pi}} \zeta(v) \\
& \times \int_{-x_{1}}^{x_{2}} \exp \left(-\frac{B^{-}}{2 A} x^{2}\right) \mathrm{d} x(1+o(1)) .
\end{aligned}
$$

Consequently, (5.3) and (5.5)-(5.7) imply that (set $\left.\bar{\zeta}(v):=D_{0} A^{3 / 2} \zeta(v) / \sqrt{B^{+}}\right)$,

$$
\begin{aligned}
& \frac{1}{D_{0} S_{1}} \mathscr{H}_{\alpha}\left(D_{0} S_{1}, D_{0} \lambda_{\varepsilon_{0}}^{-}\right)\left(\Phi\left(\left(\frac{B^{-}}{A}\right)^{1 / 2} x_{2}\right)-\Phi\left(-\left(\frac{B^{-}}{A}\right)^{1 / 2} x_{1}\right)\right) \\
& \geq \frac{\limsup _{v \rightarrow \infty} \mathbb{P}\left\{\sup _{t \in \Theta_{x_{1}, x_{2}}(v)} \inf _{s \in\left[0, \lambda_{\varepsilon_{0}} q\right]} Z(t+s)>v\right\}}{\bar{\zeta}(v)} \\
& \geq \frac{\limsup _{v \rightarrow \infty} \mathbb{P}\left\{\sup _{t \in \Theta_{x_{1}, x_{2}}(v)} \inf _{s \in[0, \lambda(v) q]} Z(t+s)>v\right\}}{\bar{\zeta}(v)} \\
& \geq \frac{\liminf _{v \rightarrow \infty} \mathbb{P}\left\{\sup _{t \in \Theta_{x_{1}, x_{2}}(v)} \inf _{s \in[0, \lambda(v) q]} Z(t+s)>v\right\}}{\bar{\zeta}(v)} \\
& \geq \frac{\liminf _{v \rightarrow \infty} \mathbb{P}\left\{\sup _{t \in \Theta_{x_{1}, x_{2}}(v)} \inf _{s \in\left[0, \lambda_{\varepsilon_{0}}^{+} q\right]} Z(t+s)>v\right\}}{\bar{\zeta}(v)} \\
& \geq \frac{1}{D_{0} S} \mathscr{H}_{\alpha}\left(D_{0} S, D_{0} \lambda_{\varepsilon_{0}}^{+}\right)\left(\Phi\left(\left(\frac{B^{+}}{A}\right)^{1 / 2} x_{2}\right)-\Phi\left(-\left(\frac{B^{+}}{A}\right)^{1 / 2} x_{1}\right)\right) \\
& -\frac{\limsup _{v \rightarrow \infty} \Sigma_{1}(v)}{\bar{\zeta}(v)} \text {. }
\end{aligned}
$$

Moreover, since

$$
\Sigma_{1}(v) \leq \sum_{-N_{1}(v) \leq k<l \leq N_{2}(v)} \mathbb{P}\left\{\sup _{t \in \Delta_{k}} Z\left(t_{0}+t\right)>v, \sup _{t \in \Delta_{l}} Z\left(t_{0}+t\right)>v\right\},
$$

similar arguments as in the proof of [23, Equations (31) and (32)] imply that

$$
\frac{\lim _{S \rightarrow \infty} \limsup _{v \rightarrow \infty} \Sigma_{1}(v)}{\bar{\zeta}(v)}=0 .
$$

Let us assume for the moment that

$$
\limsup _{S \rightarrow \infty} \frac{1}{S} \mathscr{H}_{\alpha}\left(S, D_{0} \lambda\right)>0 \text {. }
$$


First, letting $\varepsilon_{0}, \varepsilon_{1} \rightarrow 0$ and then $S, S_{1} \rightarrow \infty$, we obtain from (5.8) and the definition of $\mathscr{H}_{\alpha}$,

$$
\infty>\mathscr{H}_{\alpha} \geq \liminf _{S \rightarrow \infty} \frac{1}{S} \mathscr{H}_{\alpha}\left(S, D_{0} \lambda\right) \geq \limsup _{S \rightarrow \infty} \frac{1}{S} \mathscr{H}_{\alpha}\left(S, D_{0} \lambda\right)>0 .
$$

Furthermore, in light of (3.1) and (5.1), we have

$$
\mathbb{P}\left\{\sup _{t \geq 0} Z(t)>v\right\}=D_{0} A^{3 / 2} B^{-1 / 2} \mathscr{H}_{\alpha} \zeta(v)(1+o(1)) \quad \text { as } v \rightarrow \infty .
$$

Therefore, the claim of Theorem 2.1 follows with $\mathcal{F}_{\alpha}(\lambda) \in(0, \infty)$.

Next, we prove (5.9). Define

$$
E_{v}=\bigcup_{k}\left(\triangle_{2 k} \cap \Theta_{x_{1}, x_{2}}(v)\right), \quad N^{*}(v)=\sharp\left\{k \in \mathbb{Z}: \triangle_{2 k} \cap \Theta_{x_{1}, x_{2}}(v) \neq \varnothing\right\} .
$$

For any positive $v$,

$$
\mathbb{P}\left\{\sup _{t \in \Theta_{x_{1}, x_{2}}(v)} \inf _{s \in\left[0, \lambda_{\varepsilon_{0}}^{+} q\right]} Z(t, s)>v\right\} \geq \mathbb{P}\left\{\sup _{t \in E_{v}} \inf _{s \in\left[0, \lambda_{\varepsilon_{0}}^{+} q\right]} Z(t, s)>v\right\} .
$$

Using Bonferroni's inequality and the same arguments as in the derivation of (5.5) yields

$$
\begin{aligned}
\mathbb{P}\left\{\sup _{t \in E_{v}} \inf _{s \in\left[0, \lambda_{\varepsilon_{0}}^{+} q\right]} Z(t, s)>v\right\} \\
\quad \geq \frac{1}{2 S} \mathscr{H}_{\alpha}\left(D_{0} S, D_{0} \lambda_{\varepsilon_{0}}^{+}\right) \frac{A}{\sqrt{2 \pi}} \zeta(v) \int_{-x_{1}}^{x_{2}} \exp \left(-\frac{B^{+}}{2 A} x^{2}\right) \mathrm{d} x-\Sigma_{2}(v),
\end{aligned}
$$

where

$$
\begin{aligned}
\Sigma_{2}(v) & =\sum_{k, l \in N^{*}(v), k>l} \mathbb{P}\left\{\sup _{t \in \Delta_{2 k}} \inf _{s \in\left[0, \lambda_{\varepsilon_{0}}^{+} q\right]} Z\left(t_{0}+t+s\right)>v,\right. \\
& \left.\sup _{t \in \Delta_{2 l}} \inf _{s \in\left[0, \lambda_{\varepsilon_{0}}^{+} q\right]} Z\left(t_{0}+t+s\right)>v\right\} \\
& \leq \sum_{k, l \in N^{*}(v), k>l} \mathbb{P}\left\{\sup _{t \in \Delta_{2 k}} Z\left(t_{0}+t\right)>v, \sup _{t \in \Delta_{2 l}} Z\left(t_{0}+t\right)>v\right\} .
\end{aligned}
$$

Similar arguments as in the proof of [23, Equation (32)] show that

$$
\frac{\limsup _{v \rightarrow \infty} \Sigma_{2}(v)}{\bar{\zeta}(v)} \leq G_{3} S \sum_{k \geq 1} \exp \left(-G_{4}(k S)^{\alpha}\right)
$$

for some positive constants $G_{3}, G_{4}$. Therefore, combining (5.8) and (5.10)-(5.12), we conclude that

$$
\liminf _{S_{1} \rightarrow \infty} \frac{1}{S_{1}} \mathscr{H}_{\alpha}\left(S_{1}, D_{0} \lambda\right) \geq \frac{1}{S}\left(\frac{1}{2 D_{0}} \mathscr{H}_{\alpha}\left(D_{0} S, D_{0} \lambda\right)-G_{5} S^{2} \sum_{k \geq 1} \exp \left(-G_{4}(k S)^{\alpha}\right)\right),
$$

with some positive constant $G_{5}$. Since $\mathscr{H}_{\alpha}\left(D_{0} S, D_{0} \lambda\right)$ is positive and increasing as $S$ increases, then for sufficiently large $S$, the right-hand side in the last equation is strictly positive, thus implying (5.9). This completes the proof. 


\subsection{Proof of Theorem 3.1}

The proof is based on an application of Theorem 2.1. From (2.2), we have

$$
\mathbb{P}\left\{\tau_{u}^{*}<\infty\right\}=\mathbb{P}\left\{\sup _{t \geq 0} \inf _{s \in\left[0, S_{v}\right]} Z(t+s)>v\right\}
$$

with

$$
v=v(u)=u^{1-H / \beta}, \quad S_{v}=S_{v(u)}=T_{u} u^{-1 / \beta} \quad \text { for } u>0 .
$$

Furthermore, (3.2) implies $\lim _{v \rightarrow \infty} S_{v} / q=T \in[0, \infty)$, and

$$
\Pi(v) \leq \mathbb{P}\left\{\sup _{t \geq 0} \inf _{s \in\left[0, S_{v}\right]} Z(t+s)>v\right\} \leq \Pi(v)+\Sigma(v),
$$

where

$$
\Pi(v)=\mathbb{P}\left\{\sup _{t \in\left[t_{0}-\ln v / v, t_{0}+\ln v / v\right]} \inf _{s \in\left[0, S_{v}\right]} Z(t+s)>v\right\}, \quad \Sigma(v)=\mathbb{P}\left\{\sup _{\left|t-t_{0}\right| \geq \ln v / v} Z(t)>v\right\} .
$$

Taking $x_{1}(v)=x_{2}(v)=\ln v$ and $\lambda(v)=S_{v} / q$ in Theorem 2.1, we conclude that

$$
\begin{aligned}
\Pi(v) & =\frac{\mathcal{F}_{\alpha}\left(D_{0} T\right)}{\mathscr{H}_{\alpha}} \mathbb{P}\left\{\sup _{t \geq 0} Z(t)>v\right\}(1+o(1)) \\
& =\frac{\mathcal{F}_{\alpha}\left(D_{0} T\right)}{\mathscr{H}_{\alpha}} \mathbb{P}\left\{\tau_{u}<\infty\right\}(1+o(1)) \quad \text { as } u \rightarrow \infty .
\end{aligned}
$$

Moreover, from (5.2) we have $\Sigma(v)=o(\Pi(v))$ as $u \rightarrow \infty$, thus establishing the proof.

\subsection{Proof of Corollary 3.2}

For any $u>0$, we have

$$
\begin{aligned}
\mathbb{P}\left\{\sup _{t \geq 0} \inf _{s \in[t, t+\mathcal{T}]}\left(X_{H}(s)-c s^{\beta}\right)>u\right\} & \leq \mathbb{P}\left\{\sup _{t \geq 0}\left(X_{H}(s)-c s^{\beta}\right)>u\right\} \\
& =\mathbb{P}\left\{\tau_{u}<\infty\right\} .
\end{aligned}
$$

Furthermore, for any small positive $\varepsilon \in(0,2 H+\alpha-2 \beta)$, by the independence of $\mathcal{T}$ and $X_{H}$,

$$
\begin{aligned}
\mathbb{P}\left\{\sup _{t \geq 0} \inf _{s \in[t, t+\mathcal{T}]}\left(X_{H}(s)-c s^{\beta}\right)>u\right\} \\
\quad \geq \mathbb{P}\left\{\sup _{t \geq 0} \inf _{s \in[t, t+\mathcal{T}]}\left(X_{H}(s)-c s^{\beta}\right)>u, \mathcal{T}<u^{(2 H+\alpha-2 \beta-\varepsilon) / \alpha \beta}\right\} \\
\quad \geq \mathbb{P}\left\{\sup _{t \geq 0} \inf _{s \in\left[t, t+u^{(2 H+\alpha-2 \beta-\varepsilon) / \alpha \beta}\right]}\left(X_{H}(s)-c s^{\beta}\right)>u\right\} \mathbb{P}\left\{\mathcal{T}<u^{(2 H+\alpha-2 \beta-\varepsilon) / \alpha \beta}\right\} .
\end{aligned}
$$

Hence, the claim follows from Theorem 3.1 by letting $u \rightarrow \infty$.

\subsection{Proof of Theorem 4.1}

We use the same notation as in the proof of Theorem 3.1. For any $x \in \mathbb{R}$ and $u>0$,

$$
\begin{aligned}
\mathbb{P}\left\{\tau_{u}^{*}\right. & <\infty\} \mathbb{P}\left\{\frac{\tau_{u}^{*}-t_{0} u^{1 / \beta}}{A^{1 / 2} B^{-1 / 2} u^{H / \beta+1 / \beta-1}} \leq x \mid \tau_{u}^{*}<\infty\right\} \\
& =\mathbb{P}\left\{\tau_{u}^{*} \leq t_{0} u^{1 / \beta}+A^{1 / 2} B^{-1 / 2} x u^{H / \beta+1 / \beta-1}\right\} .
\end{aligned}
$$


Next, we focus on the asymptotics of

$$
\begin{aligned}
& \mathbb{P}\left\{\tau_{u}^{*} \leq t_{0} u^{1 / \beta}+A^{1 / 2} B^{-1 / 2} x u^{H / \beta+1 / \beta-1}\right\} \\
& =\mathbb{P}\left\{\sup _{t \in\left[0, t_{0} u^{1 / \beta}+A^{1 / 2} B^{-1 / 2} x u^{H / \beta+1 / \beta-1}\right]} \inf _{s \in\left[t, t+T_{u}\right]}\left(X_{H}(s)-c s^{\beta}\right)>u\right\} \\
& =\mathbb{P}\left\{\sup _{t \in\left[0, t_{0}+A^{1 / 2} B^{-1 / 2} x v^{-1}\right]} \inf _{s \in\left[0, S_{v}\right]} Z(t+s)>v\right\},
\end{aligned}
$$

where

$$
v=v(u)=u^{1-H / \beta}, \quad S_{v}=S_{v(u)}=T_{u} u^{-1 / \beta} \quad \text { for } u>0 .
$$

Similarly to the proof of Theorem 3.1, we have

$$
\Pi_{0}(v) \leq \mathbb{P}\left\{\sup _{t \in\left[0, t_{0}+A^{1 / 2} B^{-1 / 2} x v^{-1}\right]} \inf _{s \in\left[0, S_{v}\right]} Z(t+s)>v\right\} \leq \Pi_{0}(v)+\Sigma_{0}(v),
$$

where

$$
\begin{aligned}
& \Pi_{0}(v)=\mathbb{P}\left\{\sup _{t \in\left[t_{0}-\ln v / v, t_{0}+A^{1 / 2} B^{-1 / 2} x v^{-1}\right]} \inf _{s \in\left[0, S_{v}\right]} Z(t+s)>v\right\}, \\
& \Sigma_{0}(v)=\mathbb{P}\left\{\sup _{t \in\left[0, t_{0}-\ln v / v\right]} Z(t)>v\right\} .
\end{aligned}
$$

In light of Theorem 2.1 and (5.2), we conclude that

$$
\begin{aligned}
\mathbb{P}\left\{\tau_{u}^{*}\right. & \left.\leq t_{0} u^{1 / \beta}+A^{1 / 2} B^{-1 / 2} x u^{H / \beta+1 / \beta-1}\right\} \\
& =(1+o(1)) \frac{\mathcal{F}_{\alpha}\left(D_{0} T\right)}{\mathscr{H}_{\alpha}} \mathbb{P}\left\{\tau_{u}<\infty\right\} \Phi(x) \quad \text { as } u \rightarrow \infty .
\end{aligned}
$$

Therefore, the claim of (4.1) follows by applying Theorem 3.1. Moreover, as shown in [28, Theorem 1],

$$
\frac{\tau_{u}-t_{0} u^{1 / \beta}}{A^{1 / 2} B^{-1 / 2} u^{H / \beta+1 / \beta-1}} \mid\left(\tau_{u}<\infty\right) \stackrel{\mathrm{D}}{\rightarrow} \tilde{\mathcal{N}} \quad \text { as } u \rightarrow \infty,
$$

with $\tilde{\mathcal{N}}$ an $N(0,1)$ random variable. Consequently, by [23, Lemma 2.3], we have

$$
\left(\frac{\tau_{u}-t_{0} u^{1 / \beta}}{A^{1 / 2} B^{-1 / 2} u^{H / \beta+1 / \beta-1}}, \frac{\tau_{u}^{*}-t_{0} u^{1 / \beta}}{A^{1 / 2} B^{-1 / 2} u^{H / \beta+1 / \beta-1}}\right) \mid\left(\tau_{u}^{*}<\infty\right) \stackrel{\mathrm{D}}{\rightarrow}(\tilde{\mathcal{N}}, \tilde{\mathcal{N}}) \quad \text { as } u \rightarrow \infty,
$$

thus implying (4.2). This completes the proof.

\section{Acknowledgements}

We are grateful to the anonymous referee for his/her comments and suggestions. The authors acknowledge the generous partial support by the Swiss National Science Foundation (grant no. 200021-140633/1) and the project Risk Analysis, Ruin and Extremes (proposal no. 318984) (a Marie Curie IRSES FP7 fellowship). The first author also acknowledges partial support by Narodowe Centrum Nauki (grant no. 2013/09/B/ST1/01778). 


\section{References}

[1] Albin, J. M. P. and Choi, H. (2010). A new proof of an old result by Pickands. Electron. Commun. Prob. 15, 339-345.

[2] Asmussen, S. and Albrecher, H. (2010). Ruin Probabilities, 2nd edn. World Scientific, Hackensack, NJ.

[3] Berman, S. M. (1992). Sojourns and Extremes of Stochastic Processes. Wadsworth \& Brooks/Cole Advanced Books \& Software, Pacific Grove, CA.

[4] Chesney, M., Jeanblanc-Picqué, M. and Yor, M. (1997). Brownian excursions and Parisian barrier options. Adv. Appl. Prob. 29, 165-184.

[5] Czarna, I. (2014). Parisian ruin probability with a lower ultimate bankrupt barrier. Scand. Actuarial J. 10.1080/03461238.2014.926288.

[6] Czarna, I. And PAlmowski, Z. (2011). Ruin probability with Parisian delay for a spectrally negative Lévy risk process. J. Appl. Prob. 48, 984-1002.

[7] Czarna, I. and Palmowski, Z. (2014). Dividend problem with Parisian delay for a spectrally negative Lévy risk process. J. Optimization Theory Appl. 161, 239-256.

[8] Czarna, I. and Palmowski, Z. (2014). Parisian quasi-stationary distributions for asymmetric Lévy processes. Preprint. Available at http://arxiv.org/abs/1404.3367.

[9] Czarna, I., Palmowski, Z. And Świştek, P. (2014). Binomial discrete time ruin probability with Parisian delay. Preprint. Available at http://arxiv.org/abs/1403.7761.

[10] Dassios, A. AND Wu, S. (2008). Parisian ruin with exponential claims. Preprint. Available at http://stats.lse.ac.uk/angelos/.

[11] DȩBicki, K. (2002). Ruin probability for Gaussian integrated processes. Stoch. Process. Appl. 98, 151-174.

[12] DȩBicki, K. And Kisowski, P. (2008). A note on upper estimates for Pickands constants. Statist. Prob. Lett. 78, 2046-2051.

[13] DȩBicki, K. AND Kosiński, K. M. (2014). On the infimum attained by the reflected fractional Brownian motion. Extremes 17, 431-446.

[14] DȩBicki, K., Hashorva, E. And Ji, L. (2015). Gaussian risk models with financial constraints. Scand. Actuarial J. 6, 469-481.

[15] DȩBicKi, K., Hashorva, E. AND Ji, L. (2014). Tail asymptotics of supremum of certain Gaussian processes over threshold dependent random intervals. Extremes 17, 411-429.

[16] Dȩbicki, K., Michna, Z. ANd Rolski, T. (2003). Simulation of the asymptotic constant in some fluid models. Stoch. Models 19, 407-423.

[17] Dieker, A. B. (2005). Extremes of Gaussian processes over an infinite horizon. Stoch. Process. Appl. 115, 207-248.

[18] Dieker, A. B. AND YAKIR, B. (2014). On asymptotic constants in the theory of extremes for Gaussian processes. Bernoulli 20, 1600-1619.

[19] Embrechts, P. And Maejima, M. (2002). Selfsimilar Processes. Princeton University Press.

[20] Embrechts, P., Klüppelberg, C. And Mikosch, T. (1997). Modelling Extremal Events (Appl. Math. (New York) 33). Springer, Berlin.

[21] Griffin, P. S. (2013). Convolution equivalent Lévy processes and first passage times. Ann. Appl. Prob. 23, 1506-1543.

[22] Griffin, P. S. And Maller, R. A. (2012). Path decomposition of ruinous behavior for a general Lévy insurance risk process. Ann. Appl. Prob. 22, 1411-1449.

[23] Hashorva, E. AND Ji, L. (2014). Approximation of passage times of $\gamma$-reflected processes with FBM input. J. Appl. Prob. 51, 713-726.

[24] Hashorva, E. AND Ji, L. (2014). Extremes and first passage times of correlated fractional Brownian motions. Stoch. Models 30, 272-299.

[25] Hashorva, E. And Ji, L. (2015). Piterbarg theorems for chi-processes with trend. Extremes 18, $37-64$.

[26] Hashorva, E., Ji, L. And Piterbarg, V. I. (2013). On the supremum of $\gamma$-reflected processes with fractional Brownian motion as input. Stoch. Process. Appl. 123, 4111-4127.

[27] Hüsler, J. and Piterbarg, V. (1999). Extremes of a certain class of Gaussian processes. Stoch. Process. Appl. 83, 257-271.

[28] Hüsler, J. and Piterbarg, V. (2008). A limit theorem for the time of ruin in a Gaussian ruin problem. Stoch. Process. Appl. 118, 2014-2021.

[29] Hüsler, J. and Zhang, Y. (2008). On first and last ruin times of Gaussian processes. Statist. Prob. Lett. 78, 1230-1235.

[30] Hüsler, J., Piterbarg, V. and Rumyantseva, E. (2011). Extremes of Gaussian processes with a smooth random variance. Stoch. Process. Appl. 121, 2592-2605.

[31] KlüPPElberg, C. AND KÜHN, C. (2004). Fractional Brownian motion as a weak limit of Poisson shot noise processes-with applications to finance. Stoch. Process. Appl. 113, 333-351. 
[32] Landriault, D., Renaud, J.-F. and Zhou, X. (2014). An insurance risk model with Parisian implementation delays. Methodol. Comput. Appl. Prob. 16, 583-607.

[33] Loeffen, R., Czarna, I. And Palmowski, Z. (2013). Parisian ruin probability for spectrally negative Lévy processes. Bernoulli 19, 599-609.

[34] MandJes, M. (2007). Large Deviations for Gaussian Queues. John Wiley, Chichester.

[35] Michna, Z. (1998). Self-similar processes in collective risk theory. J. Appl. Math. Stoch. Analysis 11, 429-448.

[36] Palmowski, Z. And Świştek, P. (2014). A note on first passage probabilities of a Lévy process reflected at a general barrier. Preprint. Available at http://arxiv.org/abs/1403.1025.

[37] PIckands, J., III (1969). Upcrossing probabilities for stationary Gaussian processes. Trans. Amer. Math. Soc. 145, 51-73.

[38] Piterbarg, V. I. (1972). On the paper by J. Pickands "Upcrossing probabilities for stationary Gaussian processes". Vestnik Moskov. Univ. Ser. I Mat. Meh. 27, 25-30.

[39] Piterbarg, V. I. (1996). Asymptotic Methods in the Theory of Gaussian Processes and Fields (Trans. Math. Monogr. 148). American Mathematical Society, Providence, RI. 